\title{
Nanoporous Activated Carbons Derived from Peach Stones
}

\author{
Abhimanyu Jha, Aabhash K. Mallick, Rajeshwar M. Shrestha, Rinita Rajbhandari Joshi ${ }^{a}$ \\ Department of Applied Sciences, Pulchowk Campus, Institute of Engineering, TU, Nepal \\ ${ }^{a}$ Corresponding author: joshirinita@yahoo.com
}

Received: Dec 10, 2018

Revised: Jan 2, 2019

Accepted: Jan 4, 2019

\begin{abstract}
In this work, nanoporous activated carbons from Peach Stone powder was achieved using phosphoric acid as an activating agent and carbonization has been conducted at temperatures ranging from $400^{\circ} \mathrm{C}$ to $700^{\circ} \mathrm{C}$ using Nitrogen as inert gas in a tubular furnace, to understand the effect of the adsorption capacity with variation in temperature. Evaluation of microporosity of each of these specimens was performed by Iodine Number technique, of which the results showed a maximum amount of micropores in the carbon at the carbonization temperature of $500^{\circ} \mathrm{C}$. The morphology of the carbon samples at two extreme temperatures of $400^{\circ} \mathrm{C}$ and $700^{\circ} \mathrm{C}$ was studied using FE-SEM images, which demonstrated large amount of nanoporous in the carbon surfaces at the higher temperature. Raman Spectroscopy outcomes delineate the similar amorphous nature of the carbonaceous specimen at these temperatures with both $\mathrm{G}$ band and $\mathrm{D}$ band. These results indicate a potential to develop a good adsorbent material applicable for water purification.
\end{abstract}

Key words: Activated carbon, peach stones, Raman spectroscopy, iodine number, cyclic voltammetry

\section{Introduction}

Preparation of Activated Carbon mainly involves two steps; carbonization and activation; resulting in a wide range of processed amorphous carbon based materials with microcrystalline structure, resulting highly developed inter-particulate surface area [1]. By increasing the surface area, highly functional carbon materials can be developed which will have advance properties. These activated carbons have wide range of applications, from energy storage to water purification. Derivation of activated carbon can be from a wide range of sources but emphasis is usually given to the use of waste products. Peach (Prunus persica) seeds are used in this research for the production of carbon. These seeds are available in plenty in Nepal but rarely utilized to the extent of being marketable. Peach stones are agricultural by-products that are currently of no economic value, and have a hard lignocellulosic material shell that gives them the potential to be used as raw materials for production of granular activated carbon [4].Various methods of activation can be achieved to enhance the physical properties and performance of the carbon. This paper presents the idea of developing chemically activated carbons from peach stones and studies the properties. Activation is done using phosphoric acid as an activating agent at various temperatures ranging from $400^{\circ} \mathrm{C}$ to $700^{\circ}$ C. Characterization has been done by Iodine number, Scanning Electron Microscope (SEM) image and Raman Spectroscopy. 


\section{Experimental}

\subsection{Materials}

Peach Stones collected from the market were washed, dried and powdered using a mortar, pestle and an electric grinder. Nitrogen gas of Ultra High Pure (UHP) has been used and the chemicals used were of analytical grade.

\subsection{Preparation of Activated Carbon}

$50 \%$ concentration phosphoric acid was added to Peach Stone powder of size $300 \mu \mathrm{m}$ and stirred continuously till the right consistency of the material was achieved. This mixture was then kept in oven with temperature maintained at $100^{\circ} \mathrm{C}$ for 24 hours. The charcoal was then powdered and carbonized in a tubular furnace at $400^{\circ} \mathrm{C}$ in inert atmosphere using UHP nitrogen. The activated carbon thus obtained was then washed and filtered. Finally, the sample was sieved and activated carbon size $106 \mu \mathrm{m}$ (P-4) was preceded for characterization. Similarly the activated carbons were also prepared at temperature $500^{\circ} \mathrm{C}, 600^{\circ} \mathrm{C}$ and $700^{\circ} \mathrm{C}$ represented by P-5, P-6 and P-7.

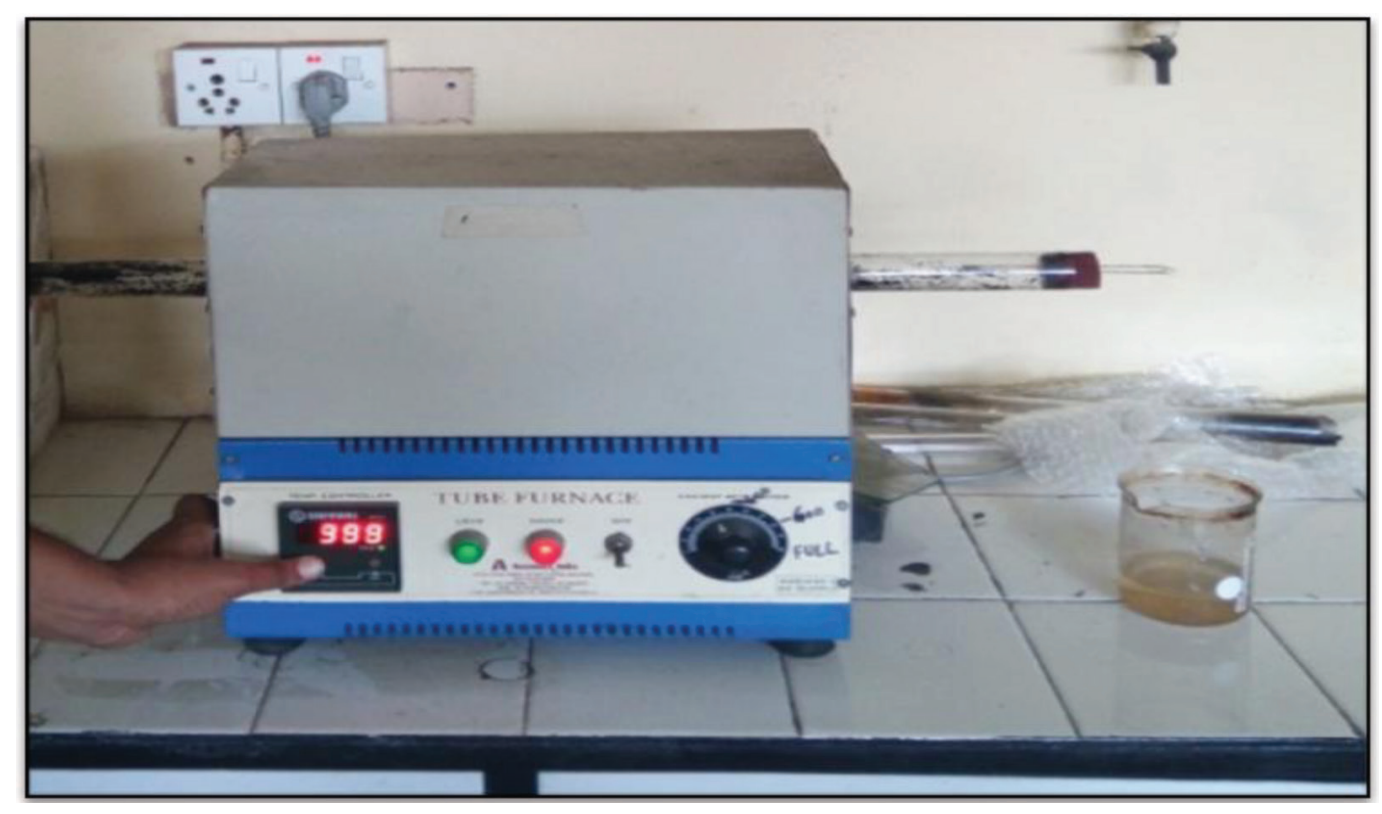

Fig. 1: Experimental Setup for Preparation of Activated Carbon in a Tube Furnace

\subsection{Determination of Iodine Number}

Iodine Number indicates the presence of microporous structures in the activated carbon. In many activated carbons the iodine number (expressed as milligrams of iodine per gram of carbon) is close to the Brunauer-Emmett-Teller (BET) surface area [3]. The Iodine Number can be calculated as.

$$
\text { Iodine number }\left(\frac{\mathrm{mg}}{\mathrm{g}}\right)=\mathrm{C} \times \text { Conversion factor }
$$


where $\mathrm{C}$ is the difference between Blank Reading and Volume of hypo solution consumed by adsorption of hypo solution by activated carbon. The Conversion Factor is determined from the equation (2) as:

$$
\text { Conversion factor }=\frac{\text { Equivalent weight of Iodine } \times \text { Normality of Iodine } \times 10}{\text { Weight of Activated carbon } \times \text { Blank reading }}
$$

\section{Results and Discussion}

\subsection{Iodine Number}

These iodine number values of the activated carbon sample do indicate increase in the micropore content from $400^{\circ} \mathrm{C}$ to $500^{\circ} \mathrm{C}$. However, the iodine number values decrease after the $500^{\circ} \mathrm{C}$ threshold when the temperature is increased, signifying that the highest number of micropores in phosphoric acid activated carbon derived from peach stones can be obtained at the carbonization temperature of $500^{\circ} \mathrm{C}$.

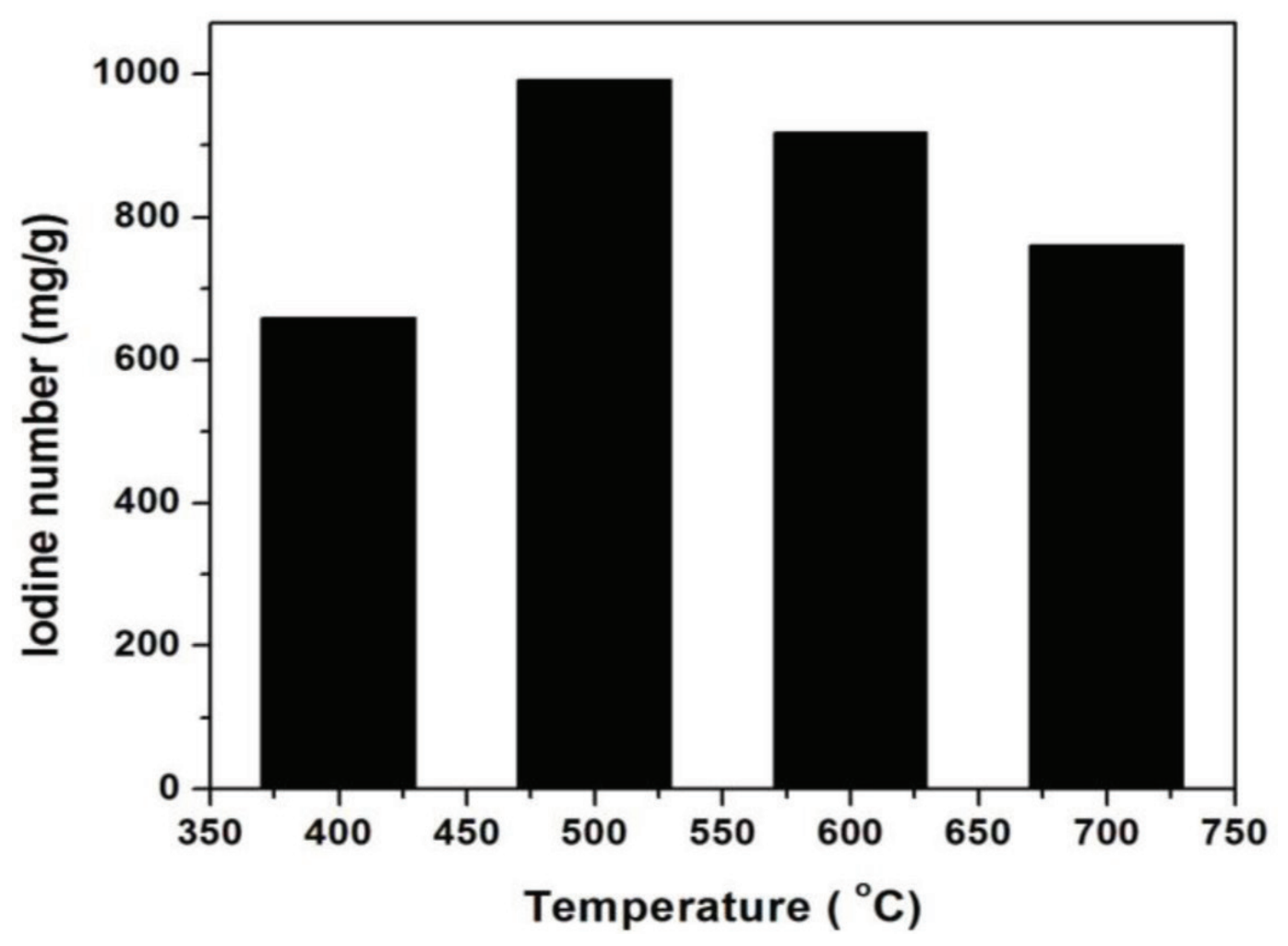

Fig. 2: Iodine number of Activated carbons

The iodine number of the activated carbon samples increases micropores from $400^{\circ} \mathrm{C}$ to $500^{\circ} \mathrm{C}$ as shown in Fig. 2. However, the iodine number decreases after $500^{\circ} \mathrm{C}$ signifying that the highest number of micropores in phosphoric acid activated carbon derived from peach stones can be obtained at the carbonization temperature of $500^{\circ} \mathrm{C}$. The decrease in Iodine number after $500^{\circ} \mathrm{C}$ may be due to the deformation of micropores. 


\subsection{Scanning Electron Microscopic images}
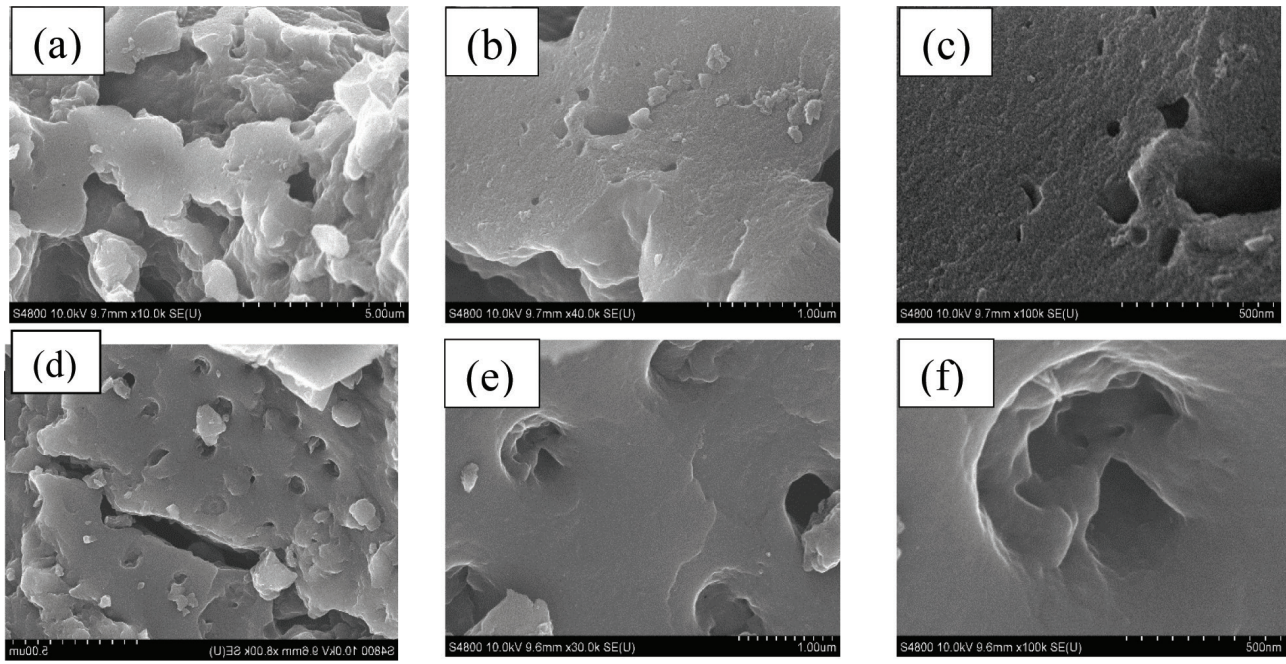

Fig. 3: FE-SEM images of P-4 Activated Carbon (a) 10K (b) 40K (c) 100K; FE-SEM Images of P-7 Activated Carbon (d) $8 \mathrm{~K}$ (e) $30 \mathrm{~K}$ (f) $100 \mathrm{~K}$

Field Emission Scanning Electron Microscope (FE-SEM) provides ultra-high resolution imaging at low accelerating voltages and small working distances [2]. Fig. 3 shows the morphological structures of the carbon samples activated at $400^{\circ} \mathrm{C}$ and $700^{\circ} \mathrm{C}$. Observation of the images at relatively close magnifications of both samples illustrates a large development of nanopores with increase in the carbonization temperatures.

\subsection{Raman Spectroscopy}

Raman Spectroscopy is extremely useful in characterization of carbonaceous materials, owing to the fact that the vibration spectra of solid compounds are much simpler than the IR spectra and are characteristic to complexes existing in the material [6]. Raman Spectroscopy has the capability to distinguish very minor changes to the morphological structure making it a invaluable tool in the characterization of carbon nanomaterials.
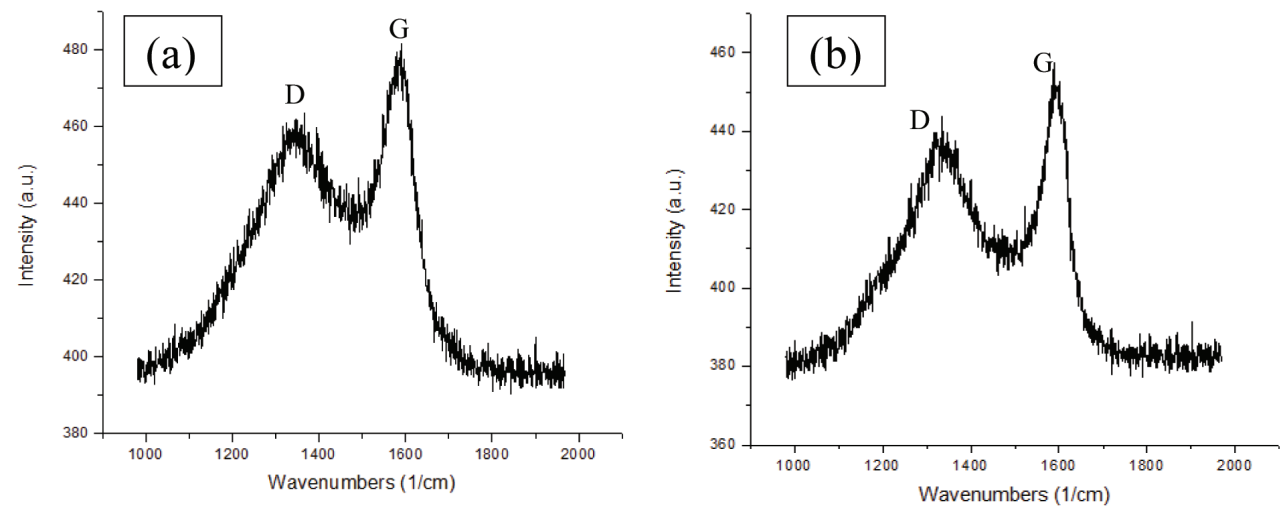

Fig. 4: Raman Spectroscopy of (a) P-4 Activated Carbon (b) P-7 Activated Carbon 
The spectrum of these activated carbons display bands which shows reduction in molecular symmetry resulting in more Raman bands being active. For both samples, the Raman Spectra appear in two frequency domains, 1300 to $1650 \mathrm{~cm}^{-1}$, corresponding to $\mathrm{G}$ and $\mathrm{D}$ bands, which is characteristic to carbonaceous compounds [6]. The band around $1580 \mathrm{~cm}^{-1}$ is known as the $\mathrm{G}$ band and the prominent band around $1350 \mathrm{~cm}^{-1}$ is known as $\mathrm{D}$ band. The $\mathrm{D}$ band is also known as disorder or defect band and in relation to the intensity of the $G$ band indicates the quality of the carbon nano-material. The graph also identifies a large vibrational frequency of atoms and illustrates the sensitivity of Raman spectroscopy to the differences in molecular morphology, corresponding to the amorphous nature of the carbon compounds.

\subsection{Cyclic Voltammetry (CV)}
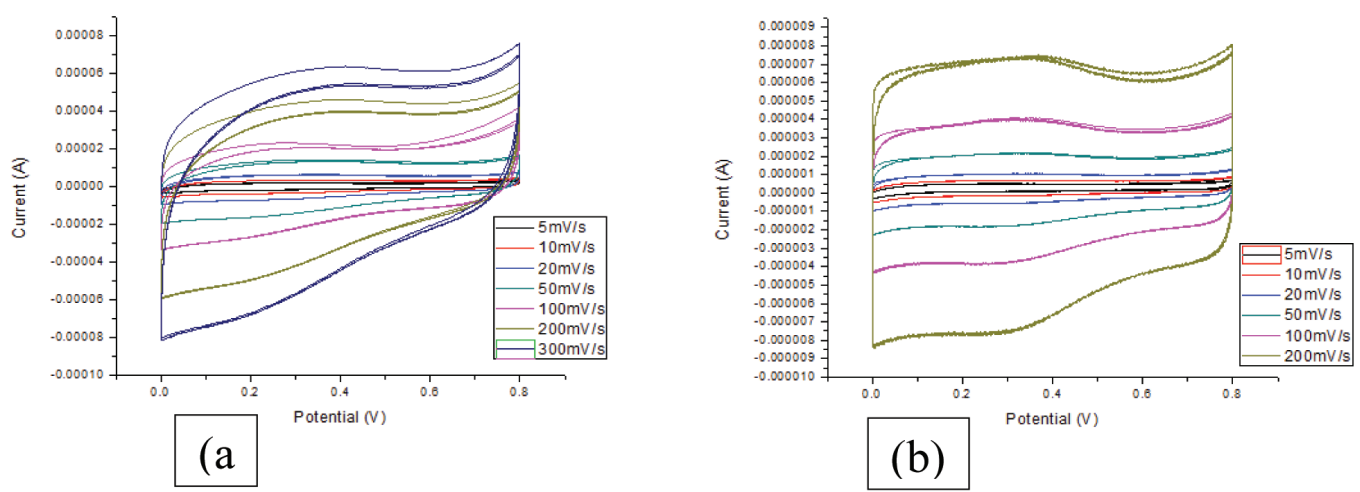

Fig. 5: Cyclic Voltammetry of (a) P-4 Activated Carbon (b) P-7 Activated Carbon

$\mathrm{CV}$ was carried out at scan rate of 5, 10,20,50,100, and $200 \mathrm{mV} / \mathrm{s}$ between the potential range of 0 to $0.8 \mathrm{~V}$. At lower scan rates a symmetrical shape is obtained about the zero current line indicating a fast reversible charge/discharge non-redux behavior as well as the consistency of adsorption and desorption of the carbon material corresponding to the symmetry at higher scan rate [5].

\section{Conclusion}

Activated carbon was successfully prepared from peach stones by activation with phosphoric acid and carbonization at various temperatures. Microporosity of the carbon specimen increases from P-4 to P-5, at which it is maximum, after which it decreases at a gradual rate with increase in temperature, as signified by the Iodine number. Raman Spectroscopy results illustrate the amorphous nature of the carbonaceous material. However, study of the FE-SEM results at the temperatures of $400^{\circ} \mathrm{C}$ and $700^{\circ} \mathrm{C}$ show a drastic increase in the nanopores of the material. Observing the FE-SEM results along with the $\mathrm{CV}$ graphs, the potential of adsorption capacity seems to be on the higher side with increase in the carbonization temperature for the activated carbon sample, which has the potential to be used as an adsorbent for removal of heavy metals, dyes and purification of water.

Acknowledgements: The authors are thankful to Prof. Dr. Bhadra P. Pokharel, Department of Applied Sciences, Pulchowk Campus for CV and Dr. Lok Kumar Shrestha from National Institute for Materials Science (NIMS), Japan for instrumental facilities. 


\section{References}

[1] Bansal RC and Goyal M (2005), Activated Carbon Adsorption, Taylor \& Francis Group, Florida, US.

[2] Che Hak CR et al. (2015), Field Emission Scanning Electron Microscope (FE-SEM) Facility in BTI, International Nuclear Information System, 47(45): 15-22.

[3] Marsh H and Rodriguez-Reinoso F (2006), Activated Carbon, Elsevier Science \& Technology Books.

[4] Masiya TT and Gudyanga FP (2009), Investigation of granular activated carbon from peach stones for gold adsorption in acidic thiourea, Hydrometallurgy Conference, The Southern African Institute of Mining and Metallurgy, 465- 474.

[5] Sim CK et al. (2015), Electrochemical Performance of Activated Carbon Derived from Treated Food-Waste, International Journal of Electrochemical Science, 10: 10157- 10172.

[6] Sisu C et al. (2016), Raman Spectroscopy Studies of Some Carbon Molecular Sieves, Digest Journal of Nanomaterials and Biostructures, 11(2): 435- 442. 